Supporting Information

Improvements in the Organic Phase Hydrothermal Synthesis of Monodisperse $\mathrm{M}_{\mathrm{x}} \mathrm{Fe}_{3-\mathrm{x}} \mathrm{O}_{4}(\mathrm{M}=\mathrm{Fe}, \mathrm{Mg}, \mathrm{Zn})$ Spinel Nanoferrites for Magnetic Fluid Hyperthermia Application

Hossein Etemadi and Paul G. Plieger*

School of Fundamental Sciences, Massey University, Private Bag 11 222, Palmerston North, New Zealand

E-mail: p.g.plieger@massey.ac.nz

Fax: 00646350 5682; Tel: 00646356 9099, extension 7825 


\section{Instrumentation and Measurements}

\section{Structural Characterization}

Powder X-ray diffraction (XRD) patterns were recorded on a Rigaku Spider X-ray diffractometer with Cu $\mathrm{K}_{\alpha}$ radiation $(\lambda=1.5406 \AA)$, at $40 \mathrm{kV}$ and $50 \mathrm{~mA}$ from $10^{\circ}$ to $80^{\circ}$ in the Bragg configuration. Indexing the XRD patterns, $d$-spacing of lattice planes ( $h k)$, lattice constant (a) and crystallite size $(D)$ were determined for all $\mathrm{M}_{\mathrm{x}} \mathrm{Fe}_{3-\mathrm{x}} \mathrm{O}_{4}(\mathrm{M}=\mathrm{Fe}$, $\mathrm{Mg}, \mathrm{Zn}$ ) nanoferrites. The $\beta$ values of the most intense X-ray peaks corresponding to (220), (311), (400), (422), (511) and (440) was calculated through Origin software with Gaussian function.

Applying Debye-Scherrer equation to the diffraction peaks together with achieved $\beta$ values, the crystallite size was calculated. Eq.1:

$$
D=\frac{K \lambda}{\beta \operatorname{Cos} \theta}(1)
$$

Where $D$ is the crystallite size, $K$ is Scherrer shape factor $(0.9), \lambda$ is the wavelength of Cu- $K_{\alpha}$ radiation $(\lambda=1.5406 \AA), \beta$ is the full-width-half-maximum (FWHM) value of the peak in radians and $\theta$ is the Bragg diffraction angle of the ( $h k)$ reflection. 
Additionally, $d$-spacing of lattice planes $(h k h)$, and lattice constant (a) values were calculated by the Bragg equation, Eq.2 and 3:

$$
\begin{aligned}
& \lambda=2 d \operatorname{Sin} \theta \\
& a=d\left(h^{2}+k^{2}+l^{2}\right)^{1 / 2}
\end{aligned}
$$

The monodispersity and diameter of the nanoferrites was probed using transmission electron microscopy (TEM, Tecnai G2 Spirit Bio-TWIN, Oregon, USA) at an acceleration voltage of $200 \mathrm{kV}$. For TEM imaging, drops of a diluted solution $(\times 100)$ of the sample was cast onto a Cu grid and dried for several minutes before imaging. Image J software was utilized for post-processing and particle size analysis. Outlines of 70-80 particles were traced manually, and the corresponding diameters with their standard deviation were given.

The hydrodynamic size $\left(d_{h}\right)$ and polydispersity index (PDI) of the particles dispersed in hexane were measured by dynamic light scattering (DLS) instrument (Zetasizer; Nano ZS, Malvern, UK) utlizing a red laser (633 nm) in backscatter mode, with an 
angle detection of $173^{\circ}$. The samples were diluted (100x), filtered through a pre-rinsed $0.2-\mu \mathrm{m}$ filter, equilibrated at $25^{\circ} \mathrm{C}$ for $1 \mathrm{~min}$ then measured three times. The mean values were reported.

\section{Chemical Composition}

The elemental atomic ratios of monodisperse nanoferrites were probed by atomic absorption spectroscopy (AAS) (GBC Scientific) and an energy dispersive spectrometer (EDS) connected to field-emission scanning electron microscopy (FESEM FEI Quanta 200).

For EDS studies, small amount of as-made nanoferrites dispersed in hexane, was sonicated for 30 min and directly mounted onto an aluminium stub using double sided tape, carbon coated (Baltec SCD 050 sputter coater) and viewed in the FEI Quanta 200 Environmental Scanning Electron Microscope (FE-SEM FEI Quanta 200). To ensure a good accuracy, several areas from different regions of each sample were scanned and spectral data was collected with a silicon EDAX unit (NJ, USA) running Genesis Spectrum software (version 5.21).

For AAS analysis, $125 \mu \mathrm{L}$ of the as-made nanoferrites dispersed in hexane were sonicated for 30 min and completely digested in concentrated $\mathrm{HNO}_{3} 70 \%: \mathrm{H}_{2} \mathrm{O}_{2} 32$ 
$\%: \mathrm{HCl} 37 \%$ with a ratio of $1: 1: 0.5 \mathrm{~mL}$ at $100{ }^{\circ} \mathrm{C}$ overnight. The solutions were finally diluted to $10 \mathrm{~mL}$ with Milli-Q water for quantification. The metal calibration standards (10-100 ppm) were prepared by diluting aliquots from a $1000 \mathrm{ppm}$ stock metal solution. Standard solutions of $10,20,30,40,60,80$ and 100 ppm were used to plot a regression curve for intensity vs. concentration. The concentrations of $\mathrm{Fe}, \mathrm{Zn}$ and Mg were measured using an AAS spectrometer (GBC Scientific) with a Fe hollow cathode lamp (252.3 nm), Zn hollow cathode lamp (279.8 nm) or Mg hollow cathode lamp $(285.2 \mathrm{~nm})$ respectively.

The proportion of inorganic cores and organic surfactants/capping agents in the sample was obtained through the heating of samples on a TA Instruments Q50 instrument. Samples were mounted to a silica sample pan and heated from room temperature up to $800^{\circ} \mathrm{C}$ under an $\mathrm{N}_{2}$ flow with a heating rate of $10{ }^{\circ} \mathrm{C} / \mathrm{min}$.

To keep the consistency for reliable information the same nanoferrites solutions were utilized for (TEM, DLS) and (EDS, AAS) measurements respectively.

The metal $(M, F e)$ and oxygen valence states of $\mathrm{M}_{x} \mathrm{Fe}_{3-\mathrm{x}} \mathrm{O}_{4}(\mathrm{M}=\mathrm{Fe}, \mathrm{Mg}, \mathrm{Zn})$ nanoferrites were probed with Kratos Axis Ultra ${ }^{\text {DLD }}$ X-ray Photoelectron Spectrometer (Kratos Analytical, Manchester, UK) equipped with a hemispherical electron energy 
analyser. Spectra were excited using monochromatic Al $\mathrm{K}_{\alpha} \mathrm{X}$-rays (1486.69 eV) with the X-ray source operating at $150 \mathrm{~W}$. The analysis area was a 300 by 700 micron spot obtained using the hybrid magnetic and electrostatic lens and the slot aperture.

Powder samples were mounted on a stainless steel holder with double sided carbon tape. A sufficient amount was used to cover the tape.

The measurements were carried out in a normal emission geometry. A charge neutralization system was used to alleviate surface charge build-up, resulting in a shift of approximately $3 \mathrm{eV}$ to lower binding energy. During curve fitting of the binding energy of $C 1 s$, the adventitious hydrocarbon on the surface was used to correct for this shift, with the saturated hydrocarbon peak set to $285 \mathrm{eV}$. Survey scans were collected with a $160 \mathrm{eV}$ pass energy, whilst core level scans were collected with a pass energy of $20 \mathrm{eV}$. The analysis chamber was at pressures in the $10^{-9}$ torr range throughout the data collection. Data analysis was performed using CasaXPS (www.casaXPS.com).

\section{Magnetic Measurements}

Magnetic characterization was recorded in a vibrating sample magnetometer (VSM) (Quantum Design P935A USA, physical property measurement system (PPMS). 
Magnetization curves as a function of the applied magnetic field ( $\mathrm{M}-\mathrm{H}$ loops) were collected from -2.00 to $2.00 \mathrm{~T}$ magnetic field at room temperature $(300 \mathrm{~K})$ to estimate saturation magnetic moment $(\mathrm{emu})$, remnant magnetizations $\left(M_{\mathrm{r}}\right)$, and coercivities $\left(H_{\mathrm{c}}\right)$ values.

Magnetization curves as a function of the temperature (M -T) also termed as zero field cooling/field cooling (ZFC/FC) curves, were recorded between 10 and $350 \mathrm{~K}$ to assess the blocking temperature and effective anisotropy constant $\left(K_{e f}\right)$ of nanoferrites. During the Zero-field-cooling (ZFC), the samples were cooled down to $10 \mathrm{~K}$ (from room temperature $\sim 300 \mathrm{~K}$ ) under a zero magnetic field. Then under an applied magnetic field of $0.02 \mathrm{~T}$, magnetization was recorded as the temperature was increased from $10 \mathrm{~K}$ to $350 \mathrm{~K}$. During FC, samples were cooled from $350 \mathrm{~K}$ to $10 \mathrm{~K}$ and the magnetization was recorded under the applied magnetic field of the same magnitude. For the XRD, TGA, VSM and XPS measurements, the nanoferrites were dispersed in hexane, precipitated with ethanol and dried under vacuum overnight prior to measurement.

Measurement of magnetically induced hyperthermic effect. 
The heating potential of synthesized nanoferrites was considered through a calorimetric non-adiabatic set up with a commercialized magnetic alternating generator (Ambrell EASYHEAT, 2.4 kW, 196 - 197 kHz). Hexane-soluble nanoferrites were put in the aqueous phase through vigorously agitation with tetramethylammonium hydroxide. The aqueous solution of nanoferrites $(5 \mathrm{mg} / \mathrm{mL})$ were sonicated for $60 \mathrm{~min}$ in order to negate the potential aggregation. Thereafter, aqueous dispersions in plastic micro centrifuge tube were placed inside a watercooled induction coil (8-turn coil, i.d. $=25 \mathrm{~mm}, \mathrm{~L}=35 \mathrm{~mm}$ ). When the coil was thermalized at $\approx 26^{\circ} \mathrm{C}$, a measurement was initiated by heating samples for 12 min with a generator operating at a frequency of $f=194.5 \mathrm{kHz}$ and field amplitude of $H=$ 114.01 $\mathrm{mT}$ at the desired current (385.6 A). The temperature elevation of the solutions was recorded in real time with a fiber optic probe (Lumasense m3300) placed in the center of the centrifuge tube. The heat dissipation rate of ferrofluids donated as specific absorption rate (SAR) was quantified by assessing the initial rate of temperature rise under a non-adiabatic approximation, Eq.4

$$
\operatorname{SAR}(W / g)=C p \frac{m_{s} d T}{m_{n} d t}
$$


Where $C_{\mathrm{p}}$ is the specific heat of the solution $\left(C_{\mathrm{p}}\right.$ water $\left.=4186 \mathrm{~W} . \mathrm{s} / \mathrm{lit} . \mathrm{K}\right), m_{\mathrm{s}}$ is the sample volume $(\mathrm{L}), m_{\mathrm{n}}$ is the mass of nanoferrite $(\mathrm{g})$, and $d T / d t$ is the initial slope of the heating curve by applying a linear fit to the change in temperature over the first $180 \mathrm{~s}(\mathrm{~K} / \mathrm{s})$. Each sample was analysed in triplicate and an average $T_{\max }$ and SAR values were recorded.

To exclude the dependence of the SAR value on the amplitude of the applied AMF and allow a direct comparison of the heating potential of our nanoferrites with the available literature data of similar ferrofluids, we utilized the intrinsic loss power (ILP) ${ }^{60}$ defined as Eq.5:

$$
I L P\left(n H m^{2} k g^{-1}\right)=\frac{S L P\left(W k g^{-1}\right)}{f(k H z) H^{2}\left(k A m^{-1}\right)}
$$

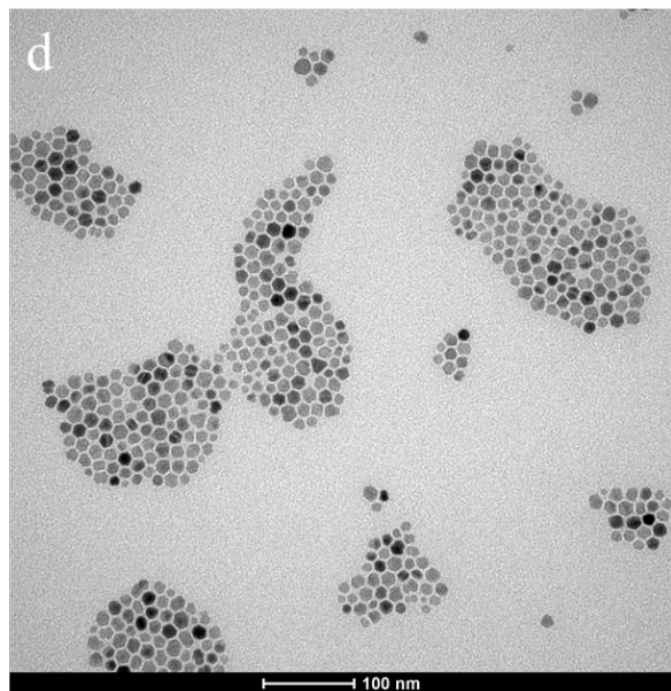



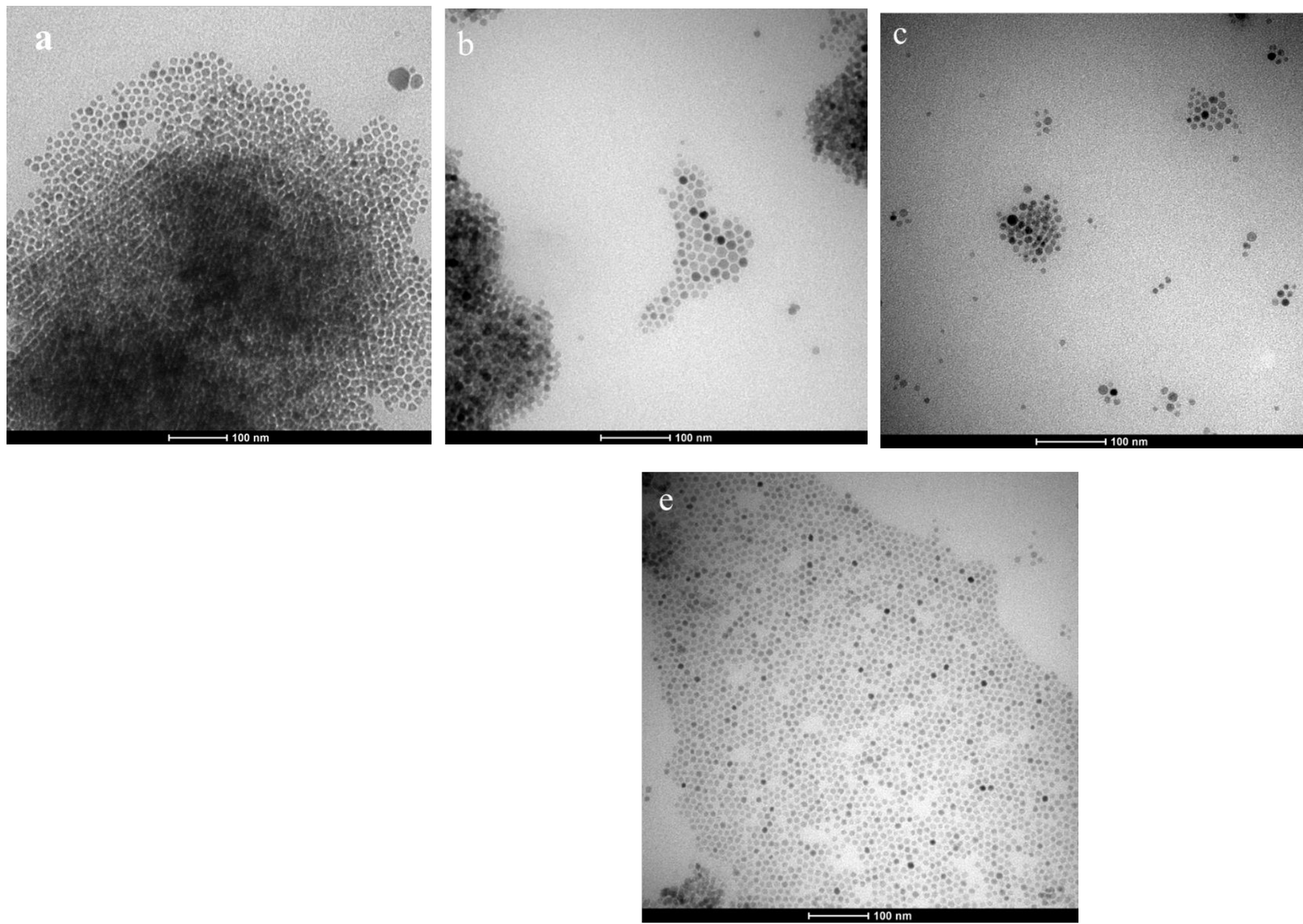

Figure S1. TEM images of the synthesised $\mathrm{Fe}_{3} \mathrm{O}_{4} \mathrm{NPs}$ with molar ratio of OA to OAm (a) 1:0 (b) 0:1 (c)

1:1; (d) $1: 4$ and (e) $1: 5 \mathrm{mmol} ; 0.5 \mathrm{mmol}$ of TOPO at $240{ }^{\circ} \mathrm{C}$ for $120 \mathrm{~min}$. 

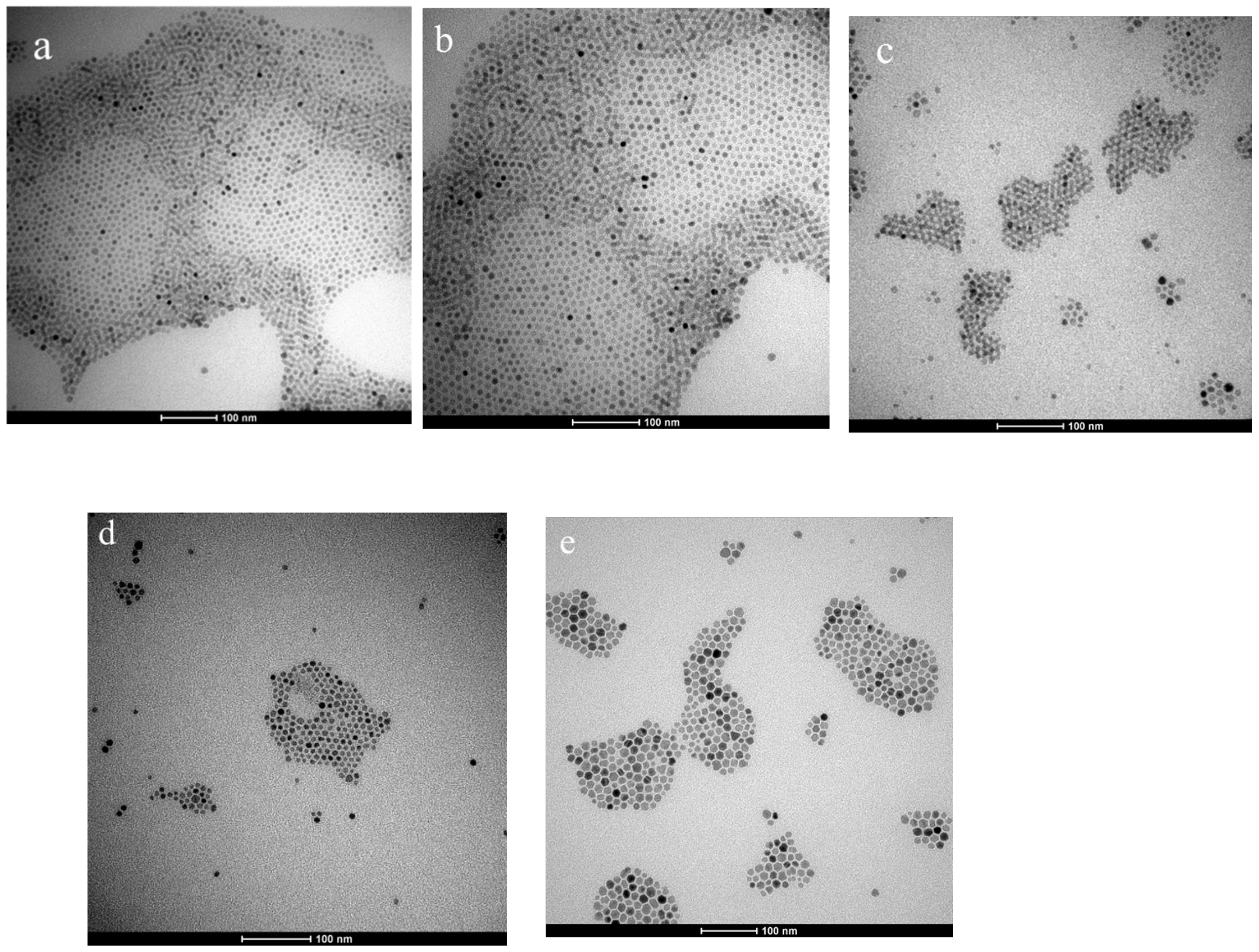

Figure S2. TEM images of the synthesized $\mathrm{Fe}_{3} \mathrm{O}_{4} \mathrm{NPs}$ with a 1:4 molar ratio of OA to OAm by different amounts of TOPO: (a) without TOPO; (b) $0.1 \mathrm{mmol}$; (c) $0.3 \mathrm{mmol}$; (d) $0.4 \mathrm{mmol}$; (e) $0.5 \mathrm{mmol}$ of TOPO at $240{ }^{\circ} \mathrm{C}$ for $120 \mathrm{~min}$. 

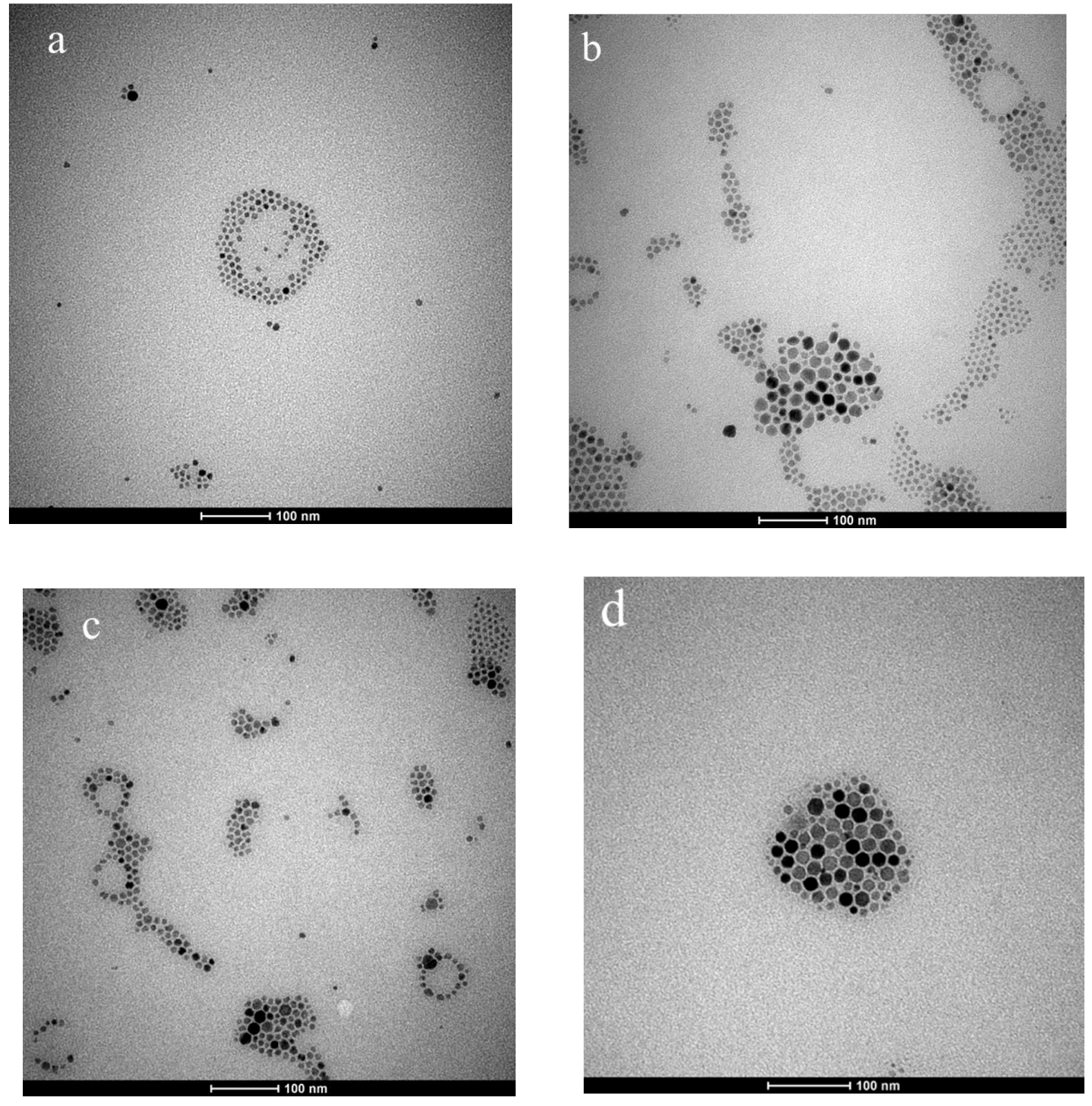

Figure S3. TEM images of the synthesised $\mathrm{Fe}_{3} \mathrm{O}_{4} \mathrm{NPs}$ with a 1:4 molar ratio of OA to OAm; TOPO 0.5 mmol at different reaction times of (a) $30 \mathrm{~min}$; (b) $60 \mathrm{~min}$ (c) $90 \mathrm{~min}$ and (d) $120 \mathrm{~min}$. 


$\begin{array}{rllll} & & \text { Size (d.nm): } & \text { \% Intensity: } & \text { St Dev (d.nm): } \\ \text { Z-Average (d.nm): } 125.5 & \text { Peak 1: } & 146.9 & 100.0 & 63.17 \\ \text { Pdl: } 0.166 & \text { Peak 2: } & 0.000 & 0.0 & 0.000 \\ \text { Intercept: } 0.919 & \text { Peak 3: } & 0.000 & 0.0 & 0.000\end{array}$

Result quality : Good

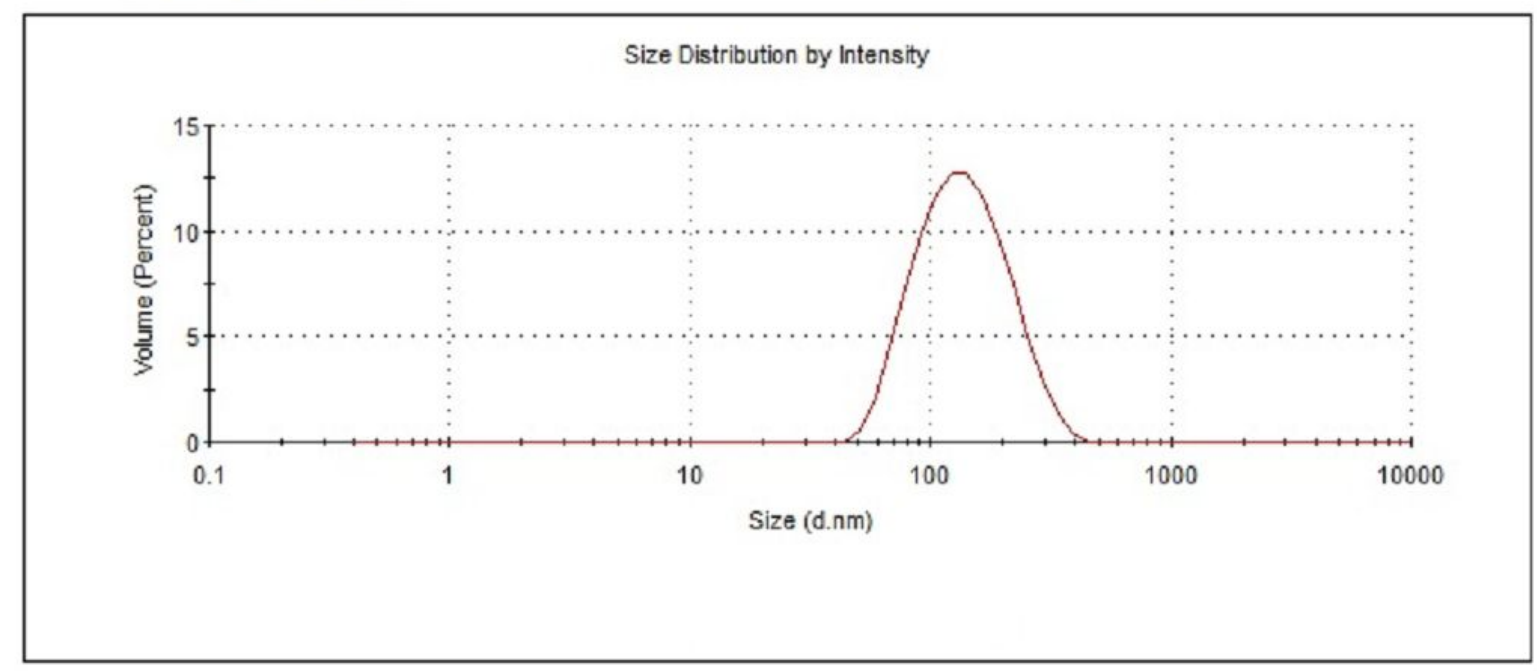

Figure S4. Volume particle size distribution (based on DLS data) of Fe1. Pdl referrers to polydispersity

\begin{tabular}{|c|c|c|c|c|c|}
\hline & & & Size (d.nm): & $\%$ Intensity: & St Dev (d.nm): \\
\hline Z-Average (d.nm): & 170.3 & Peak 1: & 161.0 & 100.0 & 46.93 \\
\hline Pdl: & 0.267 & Peak 2: & 0.000 & 0.0 & 0.000 \\
\hline Intercept: & 0.896 & Peak 3: & 0.000 & 0.0 & 0.000 \\
\hline
\end{tabular}

Result quality : Good

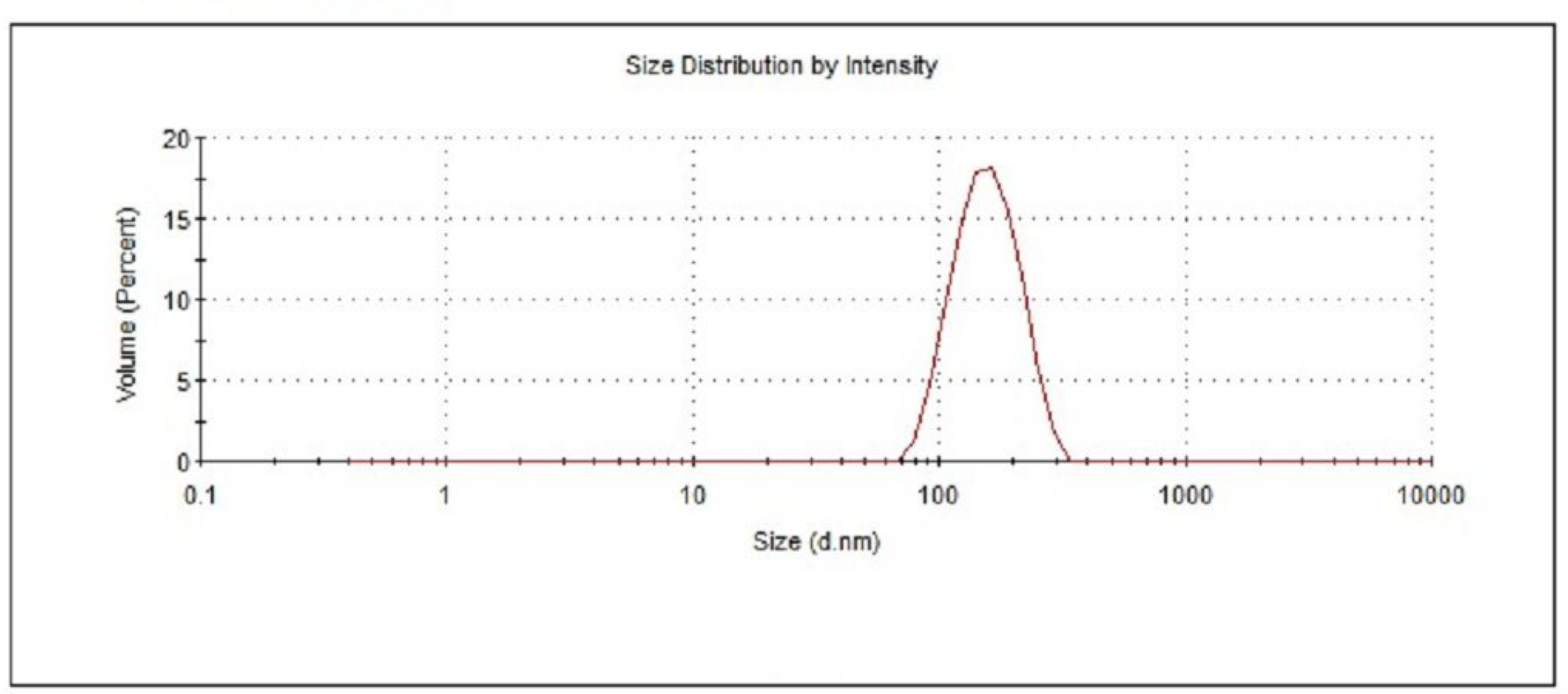

index.

Figure S5. Volume particle size distribution of Fe2. 


\begin{tabular}{|c|c|c|c|c|c|}
\hline & & & Size (d.nm): & $\%$ Intensity: & St Dev (d.nm): \\
\hline Z-Average (d.nm): & 178.9 & Peak 1: & 178.2 & 100.0 & 62.61 \\
\hline Pdl: & 0.318 & Peak 2: & 0.000 & 0.0 & 0.000 \\
\hline Intercept: & 0.911 & Peak 3: & 0.000 & 0.0 & 0.000 \\
\hline
\end{tabular}

Size Distribution by Intensity

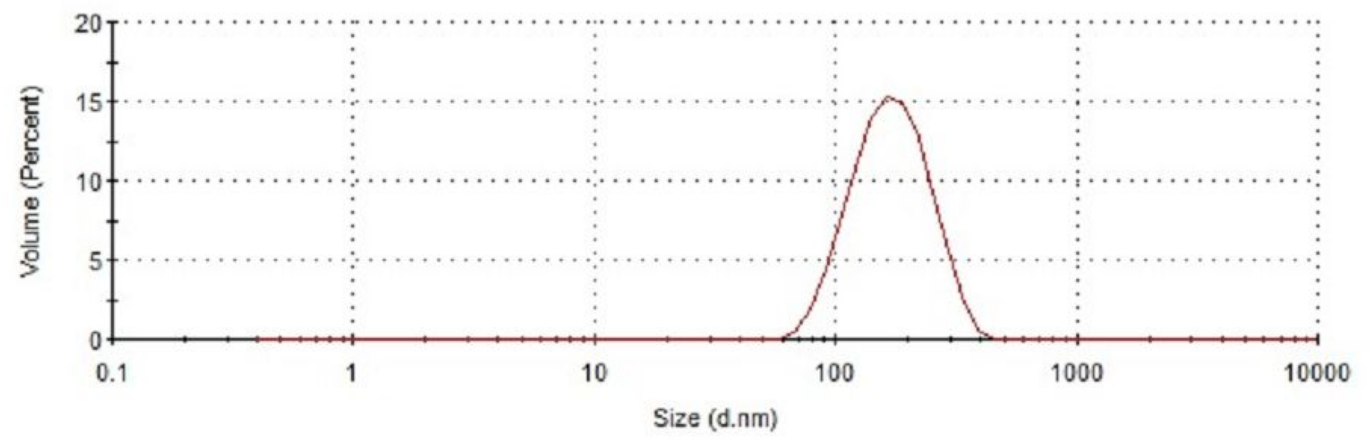

Figure S6. Volume particle size distribution of MgFe1.

Z-Average (d.nm): 189.5

Pdl: 0.247

$\begin{array}{ll} & \text { Size (d.nm) } \\ \text { Peak 1: } & 163.3 \\ \text { Peak 2: } & 0.000 \\ \text { Peak 3: } & 0.000\end{array}$

$\begin{array}{ll}\text { \% Intensity: } & \text { St Dev (d.nm): } \\ 100.0 & 40.21 \\ 0.0 & 0.000 \\ 0.0 & 0.000\end{array}$

Intercept: 0.813

Peak 3

Result quality : Refer to quality report

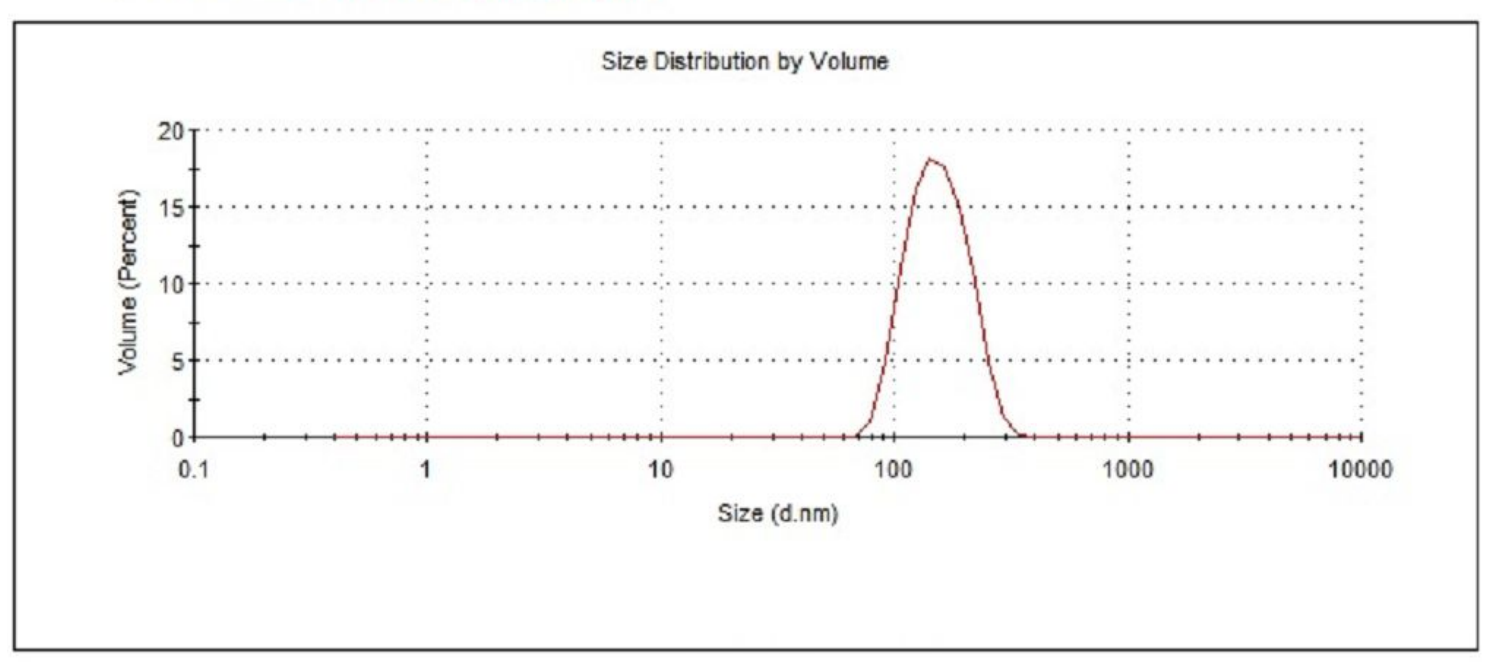

Figure S7. Volume particle size distribution of ZnFe1. 


\begin{tabular}{|c|c|c|c|c|c|}
\hline & & & Size (d.nm): & $\%$ Intensity: & St Dev (d.nm): \\
\hline Z-Average (d.nm): & 189.9 & Peak 1: & 175.2 & 100.0 & 34.12 \\
\hline Pdl: & 0.224 & Peak 2: & 0.000 & 0.0 & 0.000 \\
\hline Intercept: & 0.511 & Peak 3: & 0.000 & 0.0 & 0.000 \\
\hline
\end{tabular}

Result quality : Refer to quality report

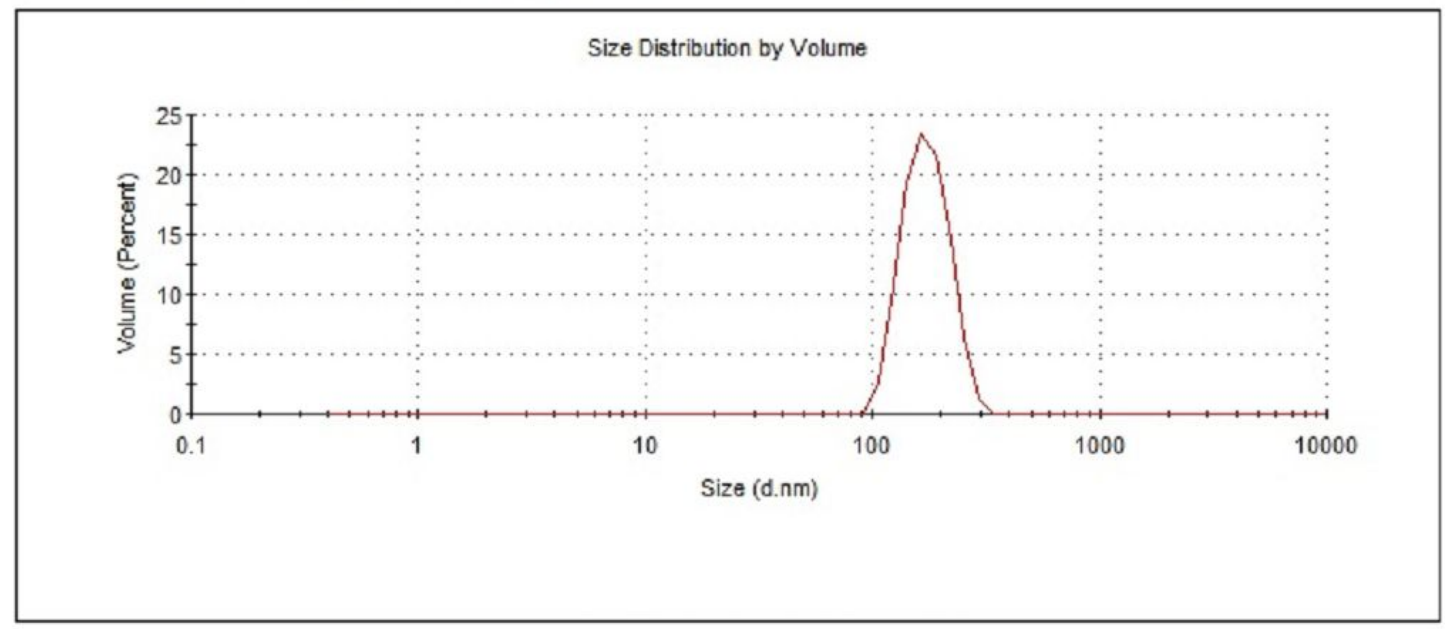

Figure S8. Volume particle size distribution of MgFe2.

$\begin{array}{rllll} & & \text { Size (d.nm): } & \text { \% Intensity: } & \text { St Dev (d.nm): } \\ \text { Z-Average (d.nm): } 214.7 & \text { Peak 1: } & 185.8 & 100.0 & 48.33 \\ \text { Pdl: } 0.259 & \text { Peak 2: } & 0.000 & 0.0 & 0.000 \\ \text { Intercept: } 0.842 & \text { Peak 3: } & 0.000 & 0.0 & 0.000\end{array}$

Result quality : Refer to quality report

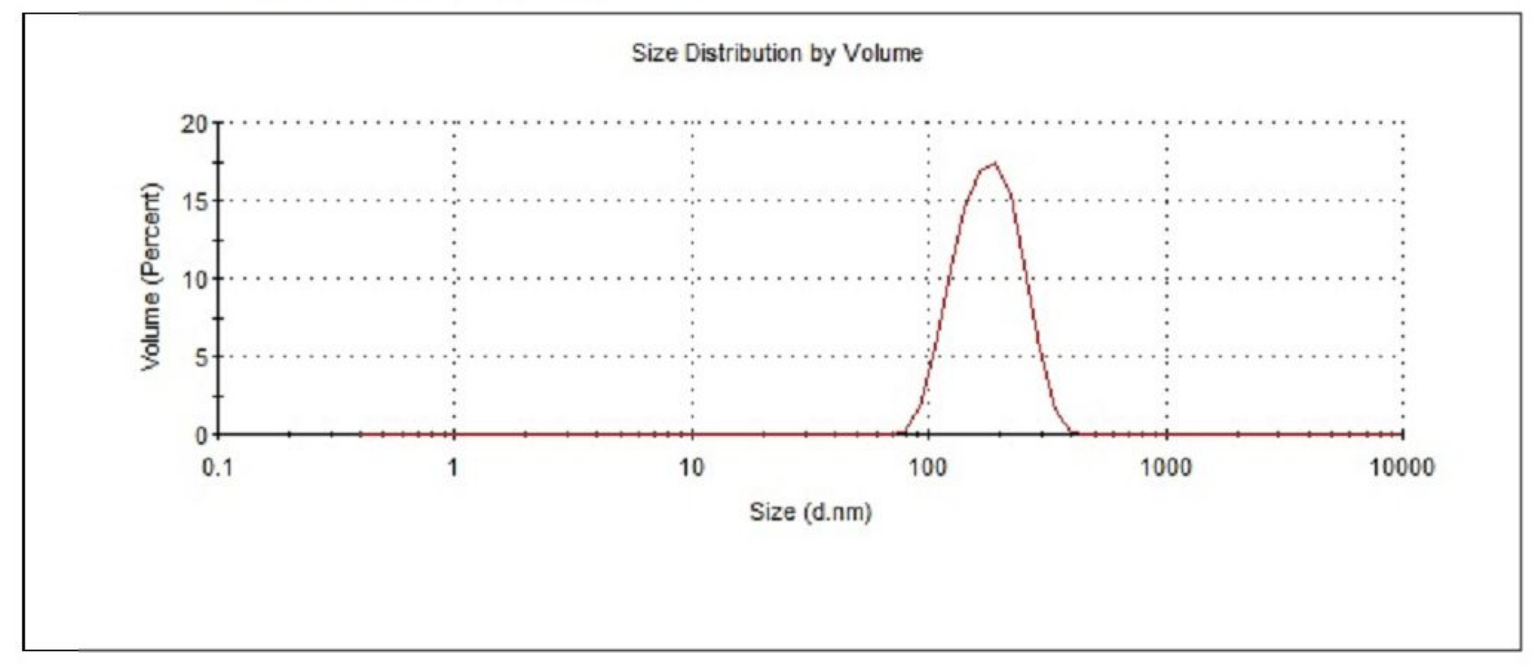

Figure S9. Volume particle size distribution of ZnFe2 


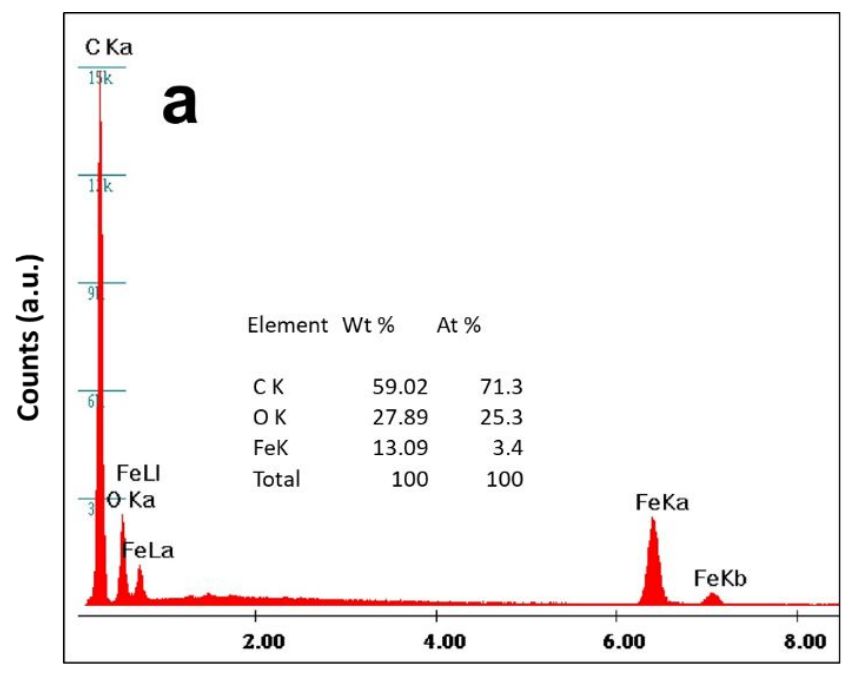

Energy (KeV)

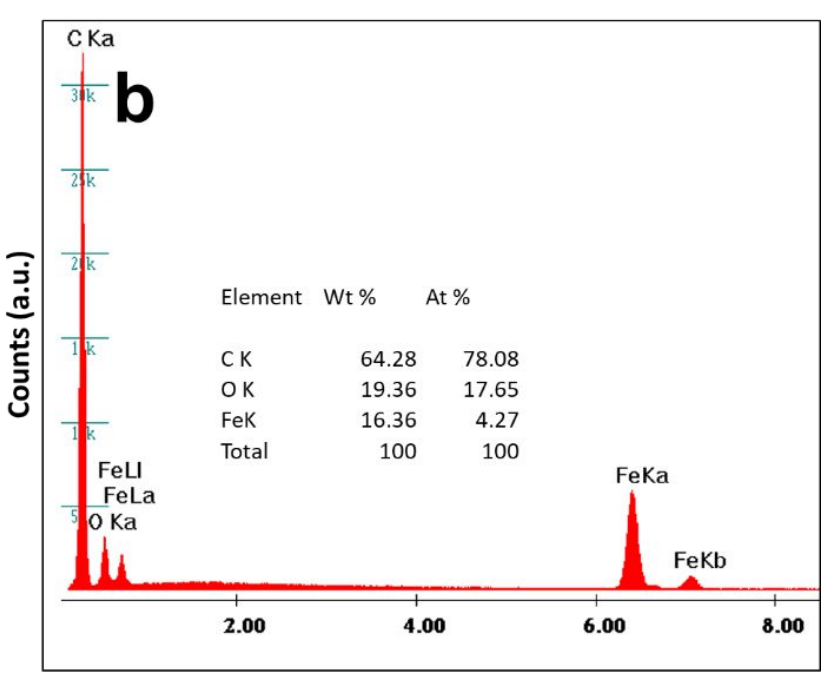

Energy (KeV)

Figure S10. EDX pattern of (a) the Fe1 and (b) the Fe2 NPs.
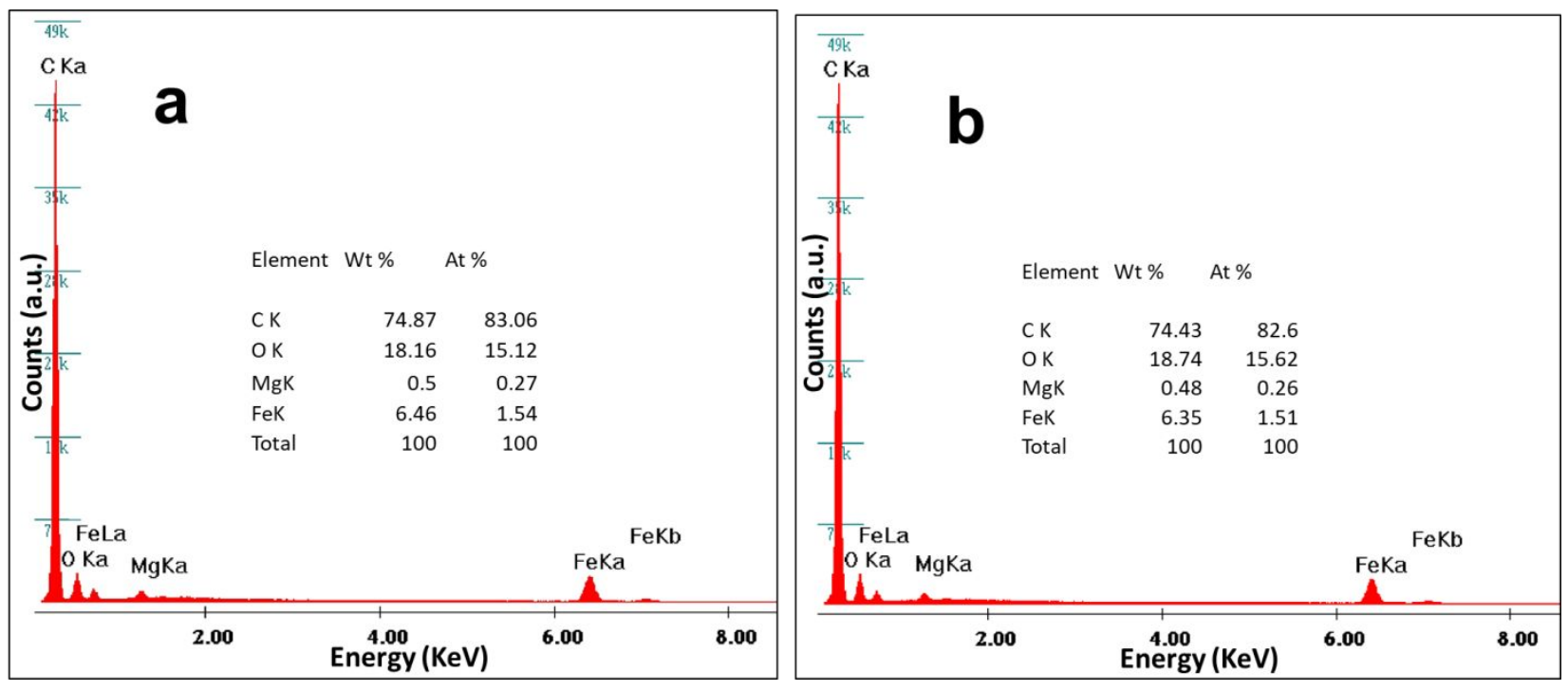

Figure S11. EDX pattern of (a) the MgFe1 and (b) the MgFe2 NPs.
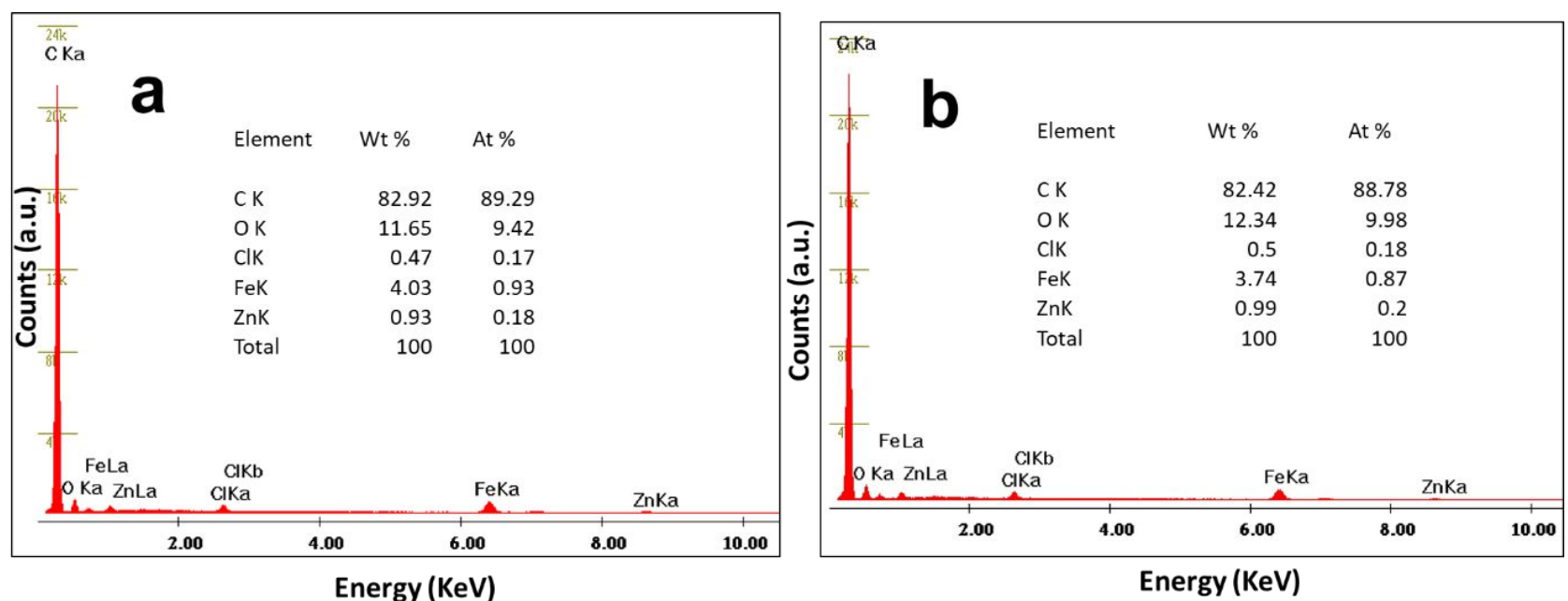
Figure S12. EDX pattern of (a) the ZnFe1 and (b) the ZnFe2 NPs.
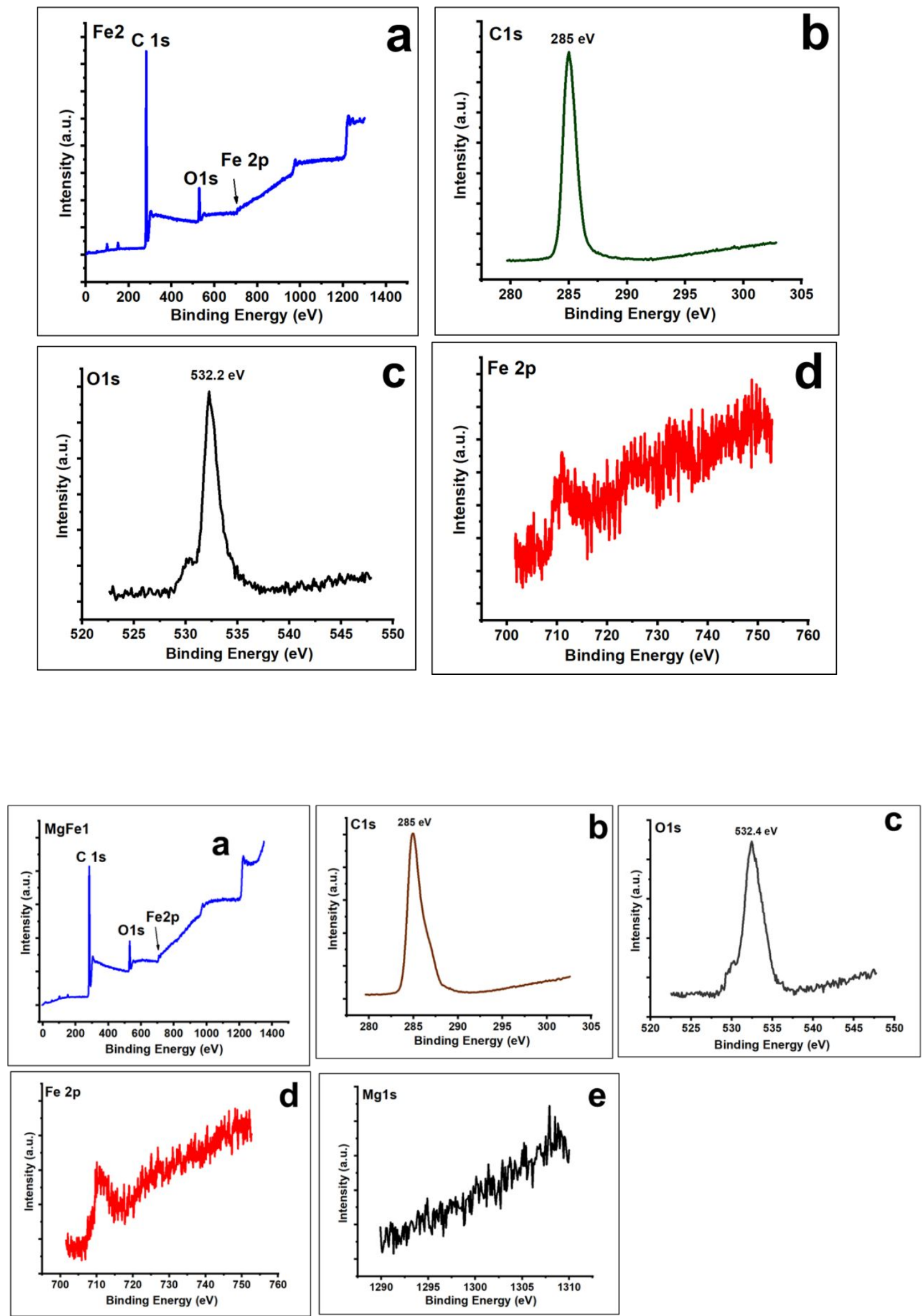

Figure S13. nanoferrite (a) survey scan (b) C $1 s$ (c) $\mathrm{O} 1 s$ and (d) Fe $2 p$ regional scans. 
Figure S14. XPS spectra of the MgFe1 nanoferrite (a) survey scan (b) C 1s (c) O 1s (d) Fe2p and (e) Mg 1s regional scans
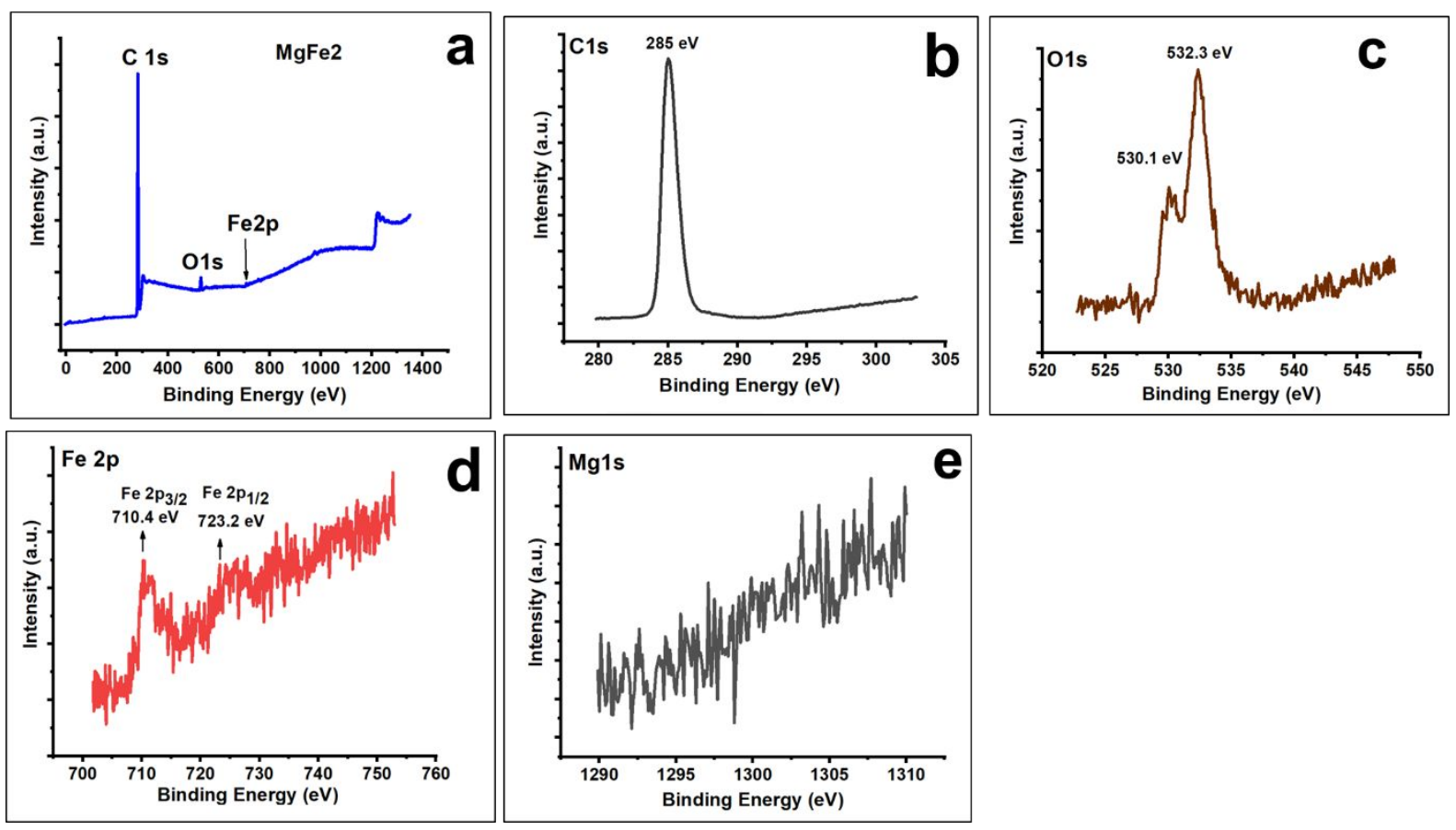
Figure S15. XPS spectra of the MgFe2 nanoferrite (a) survey scan (b) C 1s (c) O 1s (d) Fe2p and (e)
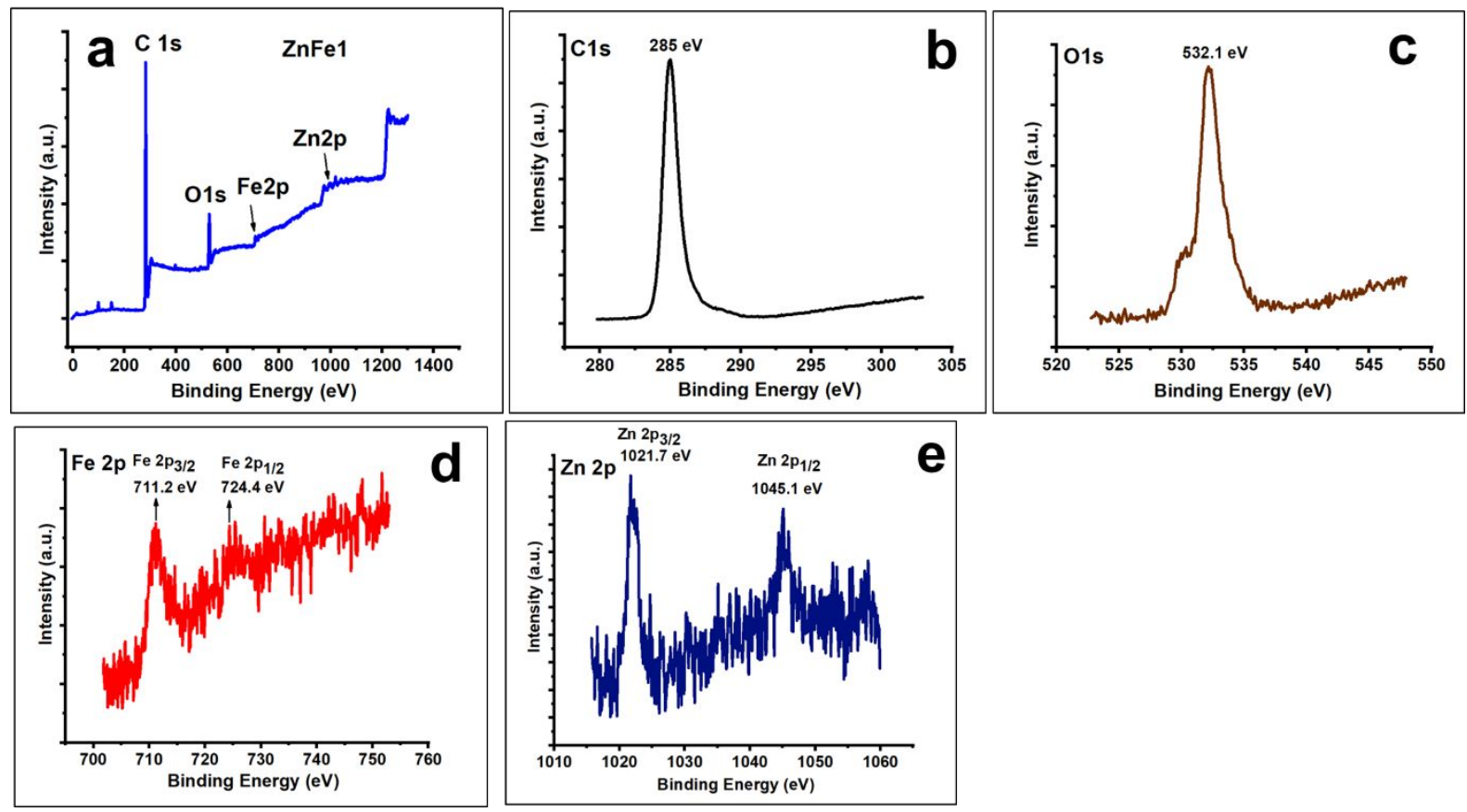

Mg 1s regional scans.

Figure S16. XPS spectra of the ZnFe1 nanoferrite (a) survey scan (b) $\mathrm{C} 1 s$ (c) $\mathrm{O} 1 s$ (d) Fe2p and (e) Zn $2 p$ regional scans. 

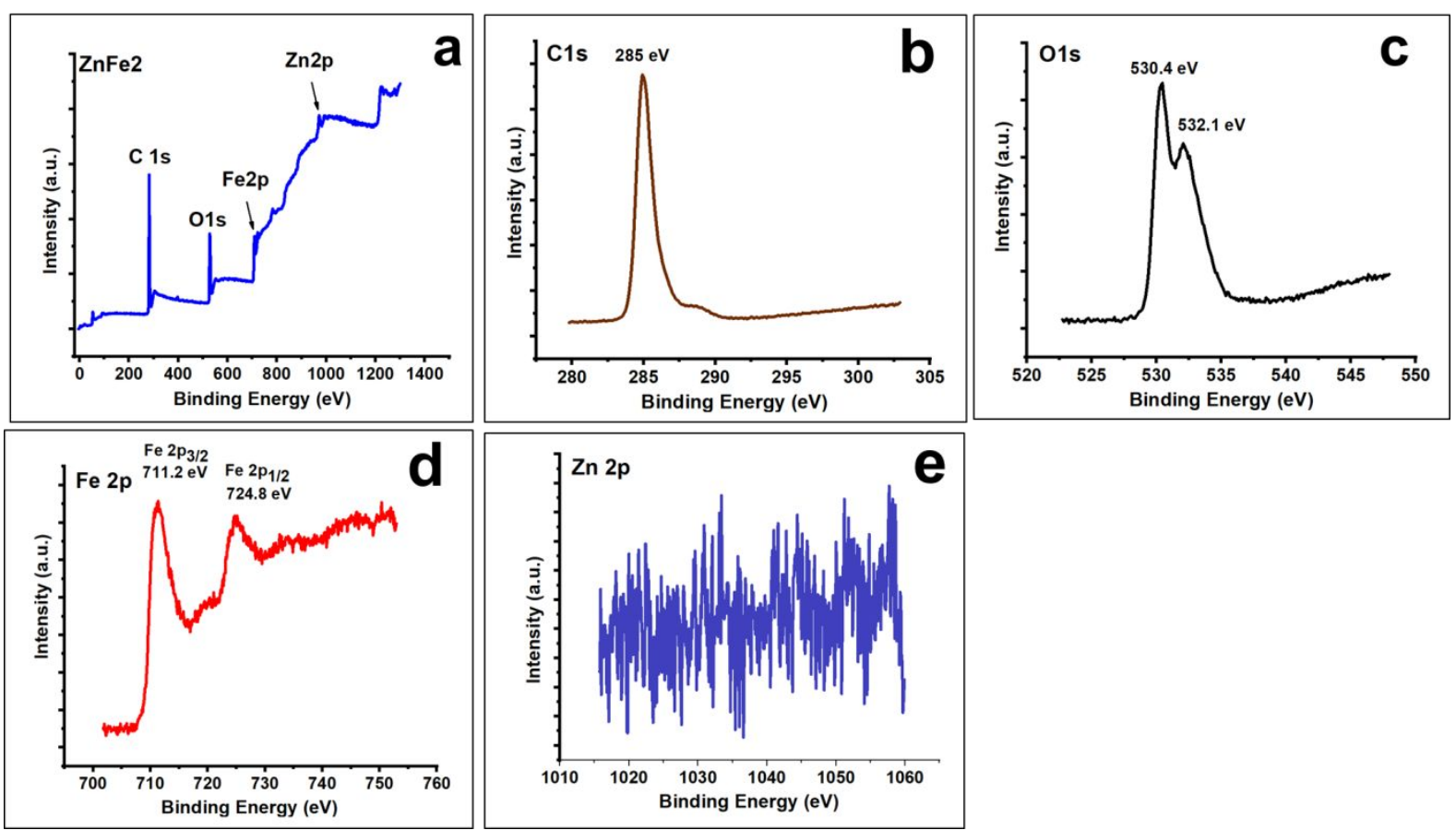

Figure S17. XPS spectra of the ZnFe2 nanoferrite (a) survey scan (b) C 1s (c) O 1s (d) Fe2p and (e) Zn2p regional scans. 